Variation of the Metallic Onset with Magnetic Field in Doped Germanium

This content has been downloaded from IOPscience. Please scroll down to see the full text. 1989 Europhys. Lett. 10269

(http://iopscience.iop.org/0295-5075/10/3/014)

View the table of contents for this issue, or go to the journal homepage for more

Download details:

IP Address: 131.215.70.231

This content was downloaded on 08/07/2014 at 18:13

Please note that terms and conditions apply. 


\title{
Variation of the Metallic Onset with Magnetic Field in Doped Germanium.
}

\author{
T. F. Rosenbaum (*), S. B. Field (**) and R. N. Bhatt $\left(^{* * *}\right)$ \\ (*) The James Franck Institute and Department of Physics \\ The University of Chicago - Chicago, IL 60637 \\ (**) Department of Physics, MIT - Cambridge, MA 02139 \\ (***) AT\&T Bell Laboratories - Murray Hill, NJ 07974
}

(received 2 May 1989; accepted in final form 21 July 1989)

PACS. 71.30 - Metal-insulator transitions.

PACS. $71.55 \mathrm{~J}$ - Localization in disordered structures.

PACS. $75.30 \mathrm{~K}$ - Magnetic phase boundaries (inc. magnetic transitions, metamagnetism, etc.).

\begin{abstract}
We demonstrate substantial magnetic-field tuning of the metal-insulator transition in $\mathrm{Ge}: \mathrm{Sb}$ for $H<20 \mathrm{kOe}$. From a fit to the conductivity in a series of samples at millikelvin temperatures in different fields to the critical form $\sigma \sim\left(n / n_{\mathrm{c}}(H)-1\right)^{\mu}$, we find that $\mu \approx 1$ independent of $H$, but that $n_{\mathrm{c}}(H)$ increases more than $25 \%$ by $H \sim 20 \mathrm{kOe}$. Moreover, $n_{\mathrm{c}}(H)-n_{\mathrm{c}}(0) \propto H^{1 / 2}$, in agreement with a weak-disorder perturbative scaling approach, but in contrast to recent experimental results in $\mathrm{Si}: \mathrm{As}$.
\end{abstract}

Doped semiconductors have served as the prototypical system for the study of the metalinsulator (MI) transition in the presence of disorder. Near the critical dopant density for the MI transition, $n_{c}$, electron diffusion effects dominate at low temperature, and electronelectron interactions are enhanced. Many experimental signatures of these localization and correlation effects have been confirmed[1]. However, there remain many unanswered questions. For example, the critical exponent of the conductivity, $\mu$, is about $1 / 2$ in uncompensated silicon [2], whereas in nominally uncompensated germanium [3] $\mu$ appears to be close to unity. Theoretically, the subject remains under active investigation [4]. Furthermore, the Hall coefficient appears to vary critically at the MI transition in doped germanium [5], but not in doped silicon [6]. In this letter, we report direct low-temperature measurements of the scaling of $n_{\mathrm{c}}$ with magnetic field, $H$, in $\mathrm{Ge}: \mathrm{Sb}$. We find further differences with results of a recently published study [7] of the magnetic tuning of the MI transition in Si:As.

Previous magnetic-field studies on InSb [8] concentrated on the qualitative phase diagram near $n_{c}$, including the high-field limit, where the impurity Bohr radius shrinks significantly, and one obtains a field-dependent Mott criterion for the MI transition. In $\mathrm{Gd}_{3-x} \mathrm{~V}_{x} \mathrm{~S}_{4}$ [9], the driving mechanism is related to the magnetic nature of the semiconductor. The closest to our work is that on the Si:As system, which is in the low-field limit. On the theoretical front, the phase diagram in a magnetic field for Anderson localization (without interaction effects) has been discussed by Shapiro[10]. Additional 
motivation for the current study comes from recent experiments which have suggested the presence of local moments in the disordered metal [11], and that the magnetic character is intertwined with the critical behavior [12].

With the larger spatial extent of its donor wavefunction, the properties of doped germanium are more easily influenced by magnetic field than those of doped silicon. Yet, in contrast to the even more field-sensitive narrow-gap semiconductors $\mathrm{Hg}_{1-x} \mathrm{Cd}_{x} \mathrm{Te}$ and $\mathrm{InSb}$, the characteristic energy scale for doped germanium is sufficiently high for millikelvin temperatures to be a good approximation to the zero temperature limit. We have studied the $T \rightarrow 0$ behavior of the conductivity of a series of $\mathrm{Ge}: \mathrm{Sb}$ samples as a function of donor density, spanning the (magnetic-field dependent) critical density for $0 \leqslant H \leqslant 20 \mathrm{kOe}$. We find that the overall behavior of the conductivity is consistent with the critical form

$$
\sigma=\sigma_{0}(H)\left[\frac{n-n_{\mathrm{c}}(H)}{n_{\mathrm{c}}(0)}\right]^{\mu} .
$$

At all fields, our data fit $\mu=1.0 \pm 0.1$ with no systematic dependence on $H$. Furthermore, we find $\sigma_{0}(H)=35 \pm 2(\Omega \mathrm{cm})^{-1}$, independent of $H$ (within our uncertainties) in the field range $H<20 \mathrm{kOe}$. At the same time, however, the critical density $n_{\mathrm{c}}(H)$, obtained by fitting the data to eq. (1) keeping $\mu=1$, rises rapidly with $H$ as

$$
n_{\mathrm{c}}(H)=n_{\mathrm{c}}(0)+\lambda \sqrt{H},
$$

and by $H=20 \mathrm{kOe}, n_{\mathrm{c}}(H)$ is over $25 \%$ larger than $n_{\mathrm{c}}(0)=(1.47 \pm 0.02) \cdot 10^{-17} \mathrm{~cm}^{-3}$. In comparison, at the highest fields studied [7] $(H \sim 200 \mathrm{kOe}), n_{\mathrm{c}}(H)$ changes by $7 \%$ in Si:As.

Single crystal boules of Ge:Sb were grown by Fritzsche [13]. Samples of typical dimensions $(9 \times 1 \times 0.4) \mathrm{mm}^{3}$ were cut from $0.4 \mathrm{~mm}$ thick slices from three of the boules and etched in CP-4 to remove any damaged surface layer. All the samples were top-loaded into a helium dilution refrigerator with direction of current flow along [111] and the axis of the transverse magnetic field along the [110] direction. Four-probe resistivity measurements

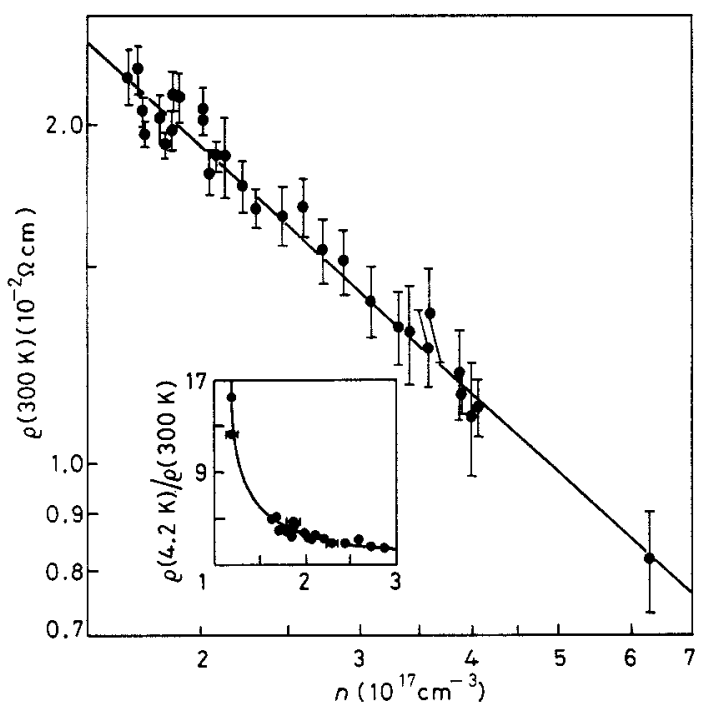

Fig. 1. - Room temperature resistivity vs. antimony donor density as determined from the Hall coefficient for Ge:Sb. The solid line is a least-squares fit, with $\rho=0.032 n^{-0.73}$. The ratio of helium to room temperature resistivities $v s$. donor density is plotted in the inset. 
were made in both the d.c. and Ohmic limits using a lock-in technique at $16 \mathrm{~Hz}$. Input power was always confined to less than $10^{-10} \mathrm{~W}$.

In order to accurately determine $n$, we fit the room temperature resistivity of a number of samples vs. donor density as determined by Hall effect measurements at $T=300 \mathrm{~K}$. The solid line in fig. 1 is a least-squares fit with $\rho \propto n^{-0.73}$, which sets our absolute donor density scale. The error bars derive from uncertainties in the sample dimensions. We eliminate this source of uncertainty by taking the ratio of sample resistivities at liquid helium and room temperatures [14], and we use those results to establish the relative density scale (see inset) more accurately very near $n_{\mathrm{c}}$ where the ratio changes rapidly.

Figure 2 shows the variation of $\sigma$ with $n$ at $T=8 \mathrm{mK}$ for various transverse $H$, illustrating the increase in the critical density with applied field. The data is reproduced within scatter at $T=50 \mathrm{mK}$, indicating that we are effectively in the $T \rightarrow 0$ limit. The solid lines are linear least-square fits to eq. (1) keeping $\mu=1$ fixed and using $\sigma_{0}(H)$ and $n_{\mathrm{c}}(H)$ as fitting parameters.

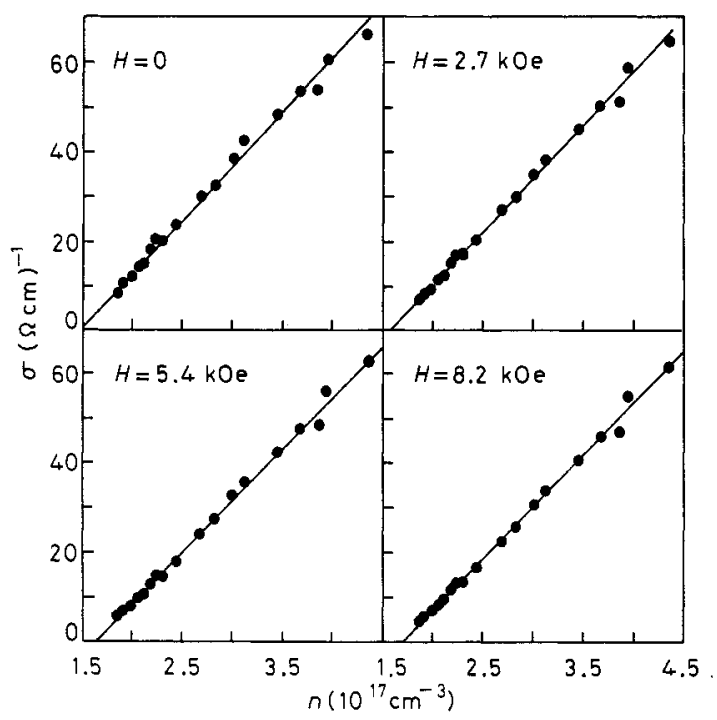

Fig. 2. - The low-temperature conductivity of a series of $\mathrm{Ge}$ :Sb samples with varying donor density in four different transverse applied fields. The solid lines are least-square fits to the critical scaling form, eq. (1), with $\mu=1$. The intercept with the abscissa determines the critical density for the metalinsulator transition, $n_{\mathrm{c}}(H) . T=8 \mathrm{mK}$.

The increase in $n_{\mathrm{c}}$ with $H$ is significantly larger than that reported [7] in Si:As. This is explained partly in terms of the larger Bohr radius and the smaller energy scale in germanium. However, we also find a different functional dependence for the change in the critical density with field. Shafarman et al. [7] fit their data to $\Delta n_{\mathrm{c}} / n_{\mathrm{c}} \propto H^{2}$, a form deduced from the field-dependent Mott criterion. With $\mu=1$, our low-field ( $\left.{ }^{1}\right)$ results $(H<20 \mathrm{kOe})$ are consistent with the form $\Delta n_{\mathrm{c}} / n_{\mathrm{c}} \propto H^{\delta}$, with $\delta=0.5$. We plot the data in fig. 3 where the solid line is a least-squares fit, $\Delta n_{\mathrm{c}} / n_{\mathrm{c}}=0.056 H^{1 / 2}$, with $H$ in kOe, which extrapolates close to the value seen by Ootuka et al. [3] for $H=40 \mathrm{kOe}$. Such a fit is motivated by a scaling form

(1) We have used $20 \mathrm{kOe}$ as a cut-off because for large fields we find the transition broadens with the development of a nonthermal tail which we believe is due to the special properties of $\mathrm{Ge:Sb}[15]$. 


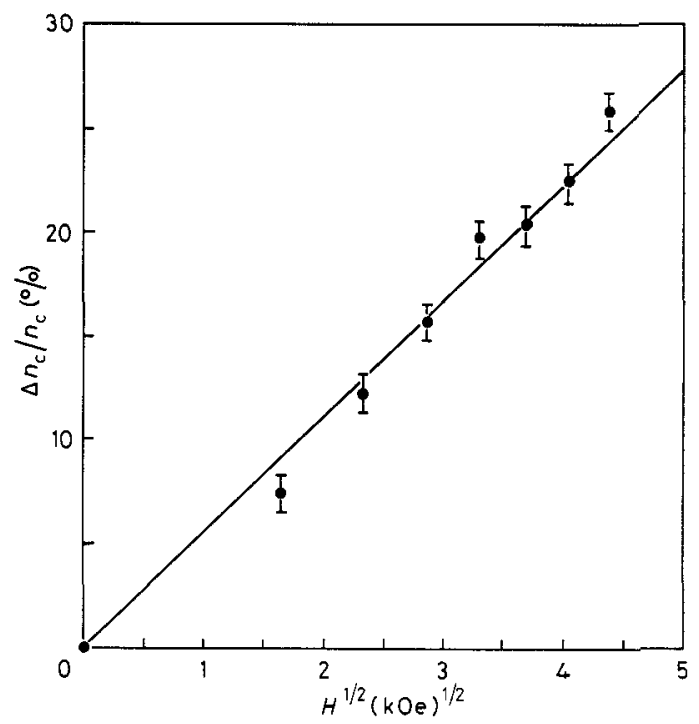

Fig. 3. - The variation of the critical density, $n_{\mathrm{c}}(H)$, normalized to $n_{\mathrm{c}}(0)$, as a function of the square root of magnetic field. The data is consistent with a weak-disorder perturbative scaling approach (see text).

for the conductivity including the crossover in magnetic field $[16,17]$ :

$$
\sigma(n, H)=\sigma_{0}\left(\frac{n}{n_{\mathrm{c}}}-1\right)^{\mu} f\left[H^{-\diamond}\left(\frac{n}{n_{\mathrm{c}}}-1\right)\right],
$$

where $n_{c}=n_{c}(0)$ is the critical density and $\mu$ is the critical exponent, both in zero field. $\delta$ and $f$ are the magnetic-field crossover exponent and function, respectively. The above form leads to a change in the critical density of the form $[16,17]$

$$
\Delta n_{\mathrm{c}}(H)=n_{\mathrm{c}}(H)-n_{\mathrm{c}}(0) \propto H^{\grave{\jmath}}
$$

In the weak-disorder regime, for noninteracting electrons, one obtains [16]

$$
\sigma(n, H)=\sigma(n, 0)+A_{1} \frac{e^{2}}{\hbar} \sqrt{\frac{e H}{c h}},
$$

so that, coupled with the scaling eq. (3), one finds that $\delta=1 / 2 \mu$ for noninteracting electrons, which is entirely consistent with our observations. The same result also follows from the observation that the argument of the scaling function is the ratio of the magnetic length, $L_{H} \sim H^{-1 / 2}$, and the localization length $\xi \sim\left(n-n_{\mathrm{c}}\right)^{-\nu}$, and that $\nu=\mu$ without interactions.

The weak-disorder perturbation theory for noninteracting electrons predicts a negative magnetoresistance, whereas we observe a positive magnetoresistance in our field range. Thus, we do not expect re-entrant behavior in $n_{\mathrm{c}}(H)$ predicted by Shapiro [10]. In fact, the magnitude and form (i.e. $H$-dependence) of the magnetoresistance $[1,18]$ is quite close to the result for interacting electrons in the weak disorder limit due to spin splitting for small 
intervalley scattering:

$$
\sigma(n, H)=\sigma(n, 0)-\frac{v e^{2} \tilde{F}_{s}}{4 \pi^{2} \hbar} \sqrt{\frac{g_{\mu} H}{2 D}},
$$

where $v$ is the number of valleys, $\tilde{F}_{\sigma}$ is a dimensionless parameter (of order unity) characterizing the Hartree interaction, and $D$ is the diffusion constant, related to the conductivity by the Einstein relation, $\sigma=e^{2}(\mathrm{~d} n / \mathrm{d} \mu) D$. For example, with $n=4 \cdot 10^{17} \mathrm{~cm}^{-3}$, $\tilde{F}_{\sigma}=1$, and using $m^{*}=0.22,\langle g\rangle=1.6, v=4$ (appropriate to $\mathrm{Ge}$ ), and $\sigma$ from experiment, eq. (6) gives $\Delta \sigma(H)=-1.3 \sqrt{H}$, where $H$ is in kOe, compared to the experimental result of $\Delta \sigma(H)=-2.0 \sqrt{H}$ for $H \leqslant 20 \mathrm{kOe}$.

Straightforward use of eq. (6), assuming $(\mathrm{d} n / \mathrm{d} \mu)$ is noncritical at $n_{\mathrm{c}}$, gives $\delta=1 / 3 \mu$. However, the scaling theory for interacting electrons involves dangerous irrelevant variables and, therefore, a conclusion based upon results in the weak-disorder limit is probably inappropriate. In any case, our experimental results are better fitted with $\mu=1$, $\delta=1 / 2$ than with any $\delta$ and $\mu$ satisfying $\delta=1 / 3 \mu$. Further, our results in the low-field regime are quite distinct from those of Shafarman et al. [7]. The contrast between the two experimental results may be due to the different method of analysis-we have fit directly the crossover behavior of metallic samples in magnetic field, whereas they have inferred it from inflections in the magnetoresistance, using samples on both sides of $n_{c}$. Other sources for the apparently dissimilar behavior could be the difference in intervalley scattering in the two systems, the strength of the spin-orbit interaction, or the possible compensation of our germanium samples.

In summary, we have studied directly the $T \rightarrow 0$ behavior of the conductivity for a series of $\mathrm{Ge}: \mathrm{Sb}$ samples in magnetic fields up to $H=20 \mathrm{kOe}$. The critical density for the metalinsulator transition is acutely sensitive to applied field, allowing magnetic tuning of the transition in laboratory fields. The variation of $n_{\mathrm{c}}$ with $H$ obtained by fitting the data with a critical form $\sigma \sim\left(n-n_{\mathrm{c}}\right)^{\mu}$, is $\Delta n_{\mathrm{c}} \sim H^{1 / 2}$. This is consistent with the variation expected from a weak-disorder perturbative scaling approach, in contrast to the interpretation of experimental results [7] on $\mathrm{Si}$ : As. The apparent discrepancies in the behavior near $n_{\mathrm{c}}$ of related doped semiconductor systems, particularly in applied magnetic field, presumably bears on the underlying mechanisms responsible for the metal-insulator transition itself.

$$
* * *
$$

We are grateful to H. FritzSCHE for generously supplying the crystals used in this experiment. RNB and TFR acknowledge the hospitality of the Aspen Center for Physics where part of this work was accomplished. The work at The University of Chicago was supported by the National Science Foundation under Grant No. DMR85-17478.

\section{REFERENCES}

[1] A general review is Lee P. A. and Ramakrishnan T. V., Rev. Mod. Phys., 57 (1985) 287.

[2] Paalanen M. A., Rosenbaum T. F., Thomas G. A. and Bhatt R. N., Phys. Rev. Lett., 48 (1982) 1248; Newman P. F. and Holcomb D. F., Phys. Rev. B, 28 (1983) 628.

[3] Ootuka Y., Matsuoka H. and KobaYashi S., in Anderson Localization, edited by T. ANDo and H. FukuYama (Springer Verlag, Berlin) 1988, p. 40.

[4] See, for example, Leroux-Hugon P., Europhys. Lett., 8 (1989) 465.

[5] Field S. B. and Rosenbaum T. F., Phys. Rev. Lett., 55 (1985) 522; Rohde M. and Miklitz H., Phys. Rev. B, 36 (1987) 7572 find similar results in amorphous $\mathrm{Bi}_{x} \mathrm{Kr}_{1-x}$. 
[6] Koon D. W. and Castner T. G., Phys. Rev. Lett., 60 (1988) 1755; Tousson E. and Ovadyahu Z., Solid State Commun., 60 (1986) 407 found similar results in $\mathrm{In}_{2} \mathrm{O}_{3}$.

[7] Shafarman W. N., Castner T. G., Brooks J. S., Martin K. P. and Naughton M. J., Phys. Rev. Lett., 56 (1986) 980.

[8] Robert J. L., Raymond A., Aulomobard R. L. and Bousquet C., Philos. Mag. B, 42 (1980) 1003; Mansfield R., Abdul-Ghader M. and Fozoomi P., Solid-State Electron., 28 (1985) 109.

[9] von Molnar S., Briggs A., Floquet J. and Remenyi G., Phys. Rev. Lett., 51 (1983) 706.

[10] Shapiro B., Philos. Mag. B, 50 (1984) 241.

[11] Ikehata S. and Kobayashi S., Solid State Commun., 36 (1985) 607; PaAlanen M. A., Sachdev S., Bhatt R. N. and RuCkenstein A. E., Phys. Rev. Lett., 57 (1986) 2061; Alloul H. and Dellouve P., Phys. Rev. Lett., 59 (1987) 578; Roy A. and Sarachik M. P., Phys. Rev. B, 37 (1988) 5531.

[12] Bhatt R. N., Phys. Scr., 14 (1986) 7; Paalanen M. A., Graebner J. E., Bhatt R. N. and SACHDEv S., Phys. Rev. Lett., 61 (1988) 578.

[13] Fritsche H., in The Metal Non-Metal Transition in Disordered Systems, edited by L. R. FRIEDMAN and D. P. TUNSTALl (SUSSP Pub., Edinburgh) 1978, p. 193, and references therein.

[14] Rosenbaum T. F., Andres K. and Thomas G. A., Solid State Commun., 35 (1980) 663.

[15] Bhatt R. N., Rosenbaum T. F. and Ramakrishnan T. V., to be published.

[16] KHMEL'Nitskil D. E. and LaRkin A. I., Solid State Commun., 39 (1981) 1069.

[17] Pfeuty P., Jasnow D. and Fisher M. E., Phys. Rev. B, 10 (1974) 2088; Singh S. and Jasnow D., Phys. Rev. B, 11 (1975) 3445.

[18] The result for the many-valley case is easily obtained by following the arguments of BHATT R. N. and LEE P. A., Solid State Commun., 48 (1983) 755. 